\title{
Open Access Transformation in Jewish Studies
}

Open Access for excellent academic publications in the field of Jewish Studies: This is the objective of the joint initiative of the Special Information Service Jewish Studies at the University Library J. C. Senckenberg in Frankfurt/Main and De Gruyter. Thanks to the support of 18 consortia partners, 8 frontlist publications from 2020 can be published as gold open access, among them this publication.

The following institutions have contributed to the funding and thus promote the open access transformation in Jewish Studies to ensure free availability for everyone:

Fachinformationsdienst Jüdische Studien, Universitätsbibliothek J. C. Senckenberg Frankfurt am Main

Staatsbibliothek zu Berlin - Preußischer Kulturbesitz

Universitätsbibliothek der Freien Universität Berlin

Universitätsbibliothek der Technischen Universität Berlin

Universitäts- und Landesbibliothek Düsseldorf

Universitätsbibliothek der Europa-Universität Viadrina Frankfurt (Oder)

Bibliothek der Vereinigten Theologischen Seminare der Georg-August-Universität Göttingen

Niedersächsische Staats- und Universitätsbibliothek Göttingen

Universitäts- und Landesbibliothek Sachsen-Anhalt

Staats- und Universitätsbibliothek Hamburg - Carl von Ossietzky

Gottfried Wilhelm Leibniz Bibliothek - Niedersächsische Landesbibliothek

Hochschule für Jüdische Studien Heidelberg

Universitäts- und Stadtbibliothek Köln

Universitätsbibliothek Mainz

Universitätsbibliothek der Ludwig-Maximilians-Universität München

Universitäts- und Landesbibliothek Münster

Herzog August Bibliothek Wolfenbüttel

Universitätsbibliothek Wuppertal 
